\title{
Case-finding for COPD in primary care: a qualitative study of patients' perspectives
}

This article was published in the following Dove Press journal: International Journal of COPD

\author{
Alexandra Enocson \\ Kate Jolly \\ Rachel Elizabeth Jordan \\ David A Fitzmaurice \\ SM Greenfield \\ Peymane Adab

\section{On behalf of the BLISS} \\ research team
}

Institute of Applied Health Research, University of Birmingham, Edgbaston, Birmingham, West Midlands, UK
Correspondence: SM Greenfield Institute of Applied Health Research, University of Birmingham, Edgbaston, Birmingham, West Midlands BI5 2TT, UK Tel +44 I2I 4I46493

Email s.m.greenfield@bham.ac.uk
Background: COPD is a leading cause of morbidity and mortality, yet it remains largely underdiagnosed. Case-finding is encouraged by many professionals, but there is a lack of information on the patients' views and perspectives.

Patients and methods: Semistructured interviews were conducted with adults, aged 40 years or older with a history of smoking, who were eligible and invited for case-finding for COPD as a part of a large UK primary care trial. Patients, including those who consented or declined participation and those with and without COPD after screening, were interviewed. Interviews were transcribed and analyzed using the framework method.

Results: The 43 interviews revealed the following two main categories of themes: patients' views on COPD case-finding and barriers to case-finding. Overall, case-finding was deemed important and beneficial. Participants highlighted the need for screening activities to be convenient for patients but perceived that general practitioners (GPs) lacked the time and accessing appointments was difficult. Desire for a health check among symptomatic patients facilitated participation in case-finding. Psychological barriers to engagement included denial of ill health or failure to recognize symptoms, fear of the "test", and lung symptoms being low on the hierarchy of patient health complaints. Mechanical barriers included providing care for another person (and therefore being too busy), being unable to access GP appointments, and lacking feedback of spirometry results or communication of the diagnosis.

Conclusion: Patient engagement with case-finding may be limited by denial or lack of recognition of symptoms and physical barriers to attendance. Increasing public awareness of COPD risk factors and early symptoms may enhance case-finding.

Keywords: COPD, screening, qualitative research, primary care

\section{Introduction}

COPD has high morbidity and mortality with a growing financial impact upon health care systems. ${ }^{1,2}$ At least half of individuals with clinical COPD remain undiagnosed until significant damage has occurred. ${ }^{3,4}$ This is partly due to the slow and varied pattern of disease progression and patients' lack of recognition of disease onset. ${ }^{4-6}$ Several organizations and professional bodies encourage COPD case-finding. ${ }^{7,9}$ The rationale for early detection is that earlier treatment and better lifestyle choices, such as smoking cessation, will lead to improved long-term prognosis. However, the US Preventive Services Taskforce (USPSTF) and UK National Screening Committee (UK NSC) do not recommend active case-finding at present, because evidence for several aspects of the process is lacking. ${ }^{8,10}$ One area where further information is needed relates to obtaining patients' perspectives on the acceptability of case-finding, screening processes, possible barriers and facilitators that may affect uptake, and long-term outcomes of case-finding. ${ }^{8,11}$

A few studies have explored the views of patients on early symptoms of COPD, reasons for delayed diagnosis and late symptom presentation, and the perceptions of 
COPD among patients at diagnosis., ${ }^{4,69}$ Qualitative interviews were conducted with people who were either newly diagnosed or at risk of COPD, as part of US multimethod study aimed at developing a new screening questionnaire for identifying COPD. However, participants had not experienced any aspect of the screening process, and although themes relating to symptoms and warning signals for COPD were identified, their views of the case-finding process and outcomes could not be sought. ${ }^{6}$ Another study in Tasmania explored the reasons for the lack of early diagnosis of COPD in primary care. The investigators interviewed patients with COPD, as well as those with a history of recurrent respiratory prescriptions, and found that misperceptions and difficulties with disease labels were common and patients expressed frustration with the delay of a diagnosis and its implications by clinicians. ${ }^{4}$ Denial of the diagnosis and lack of recognition of symptoms were other themes that emerged from a UK study that explored the views of recently diagnosed COPD patients. ${ }^{12}$ None of these previous studies included people who had been invited or experienced the process of case-finding, or sought the views of patients on the principle or process of case-finding. It is important to understand the patient's perspective to ensure whether screening is acceptable and to identify the needs of patients, so they can make informed decisions, in relation to screening.

In this study, we recruited patients who were invited to take part in a primary care COPD case-finding trial, TargetCOPD, to explore their views and attitudes about COPD case-finding in general and on the processes involved. ${ }^{13}$

\section{Methods}

This qualitative study was nested within the TargetCOPD trial, which compared active case-finding with routine care, in terms of yield (number of new cases of undiagnosed COPD detected). ${ }^{13}$ For the trial, eligible subjects were between 40 and 79 years, with a smoking history, and no prior diagnosis of COPD. Patients in the active case-finding arm received a screening questionnaire through their general practice, and those reporting relevant respiratory symptoms were then invited for diagnostic spirometry. Those with airflow obstruction on spirometry fulfilled the study criterion of COPD and had their results sent to their general practice.

The study was approved by the National Research Ethics Service, Solihull, West Midlands, UK (11/WM/0403).

\section{Recruitment for interviews}

Given the focus for this study was to explore the experiences of those who were eligible and invited for case-finding, we sought to invite people with different responses to the invitation and different outcomes from screening and the screening process. This study recruited patients from the intervention arm of the trial, including those who did or did not attend (DNA) for case-finding and those who were screened positive or negative. We interviewed subjects of different age, gender, smoking and employment statuses to ensure we obtained a range of perspectives.

We invited patients from different parts of the casefinding process with the aim of eliciting a range of views on the key steps of case-finding. A total of 751 eligible patients, from the first three general practices in the intervention arm of the trial, with the largest number of patients, were invited for interview. These practices were chosen because at recruitment at least 6 months had passed from the time of screening (to allow sufficient time for those with a new diagnosis to have been informed). From the 58 patients who were willing to be interviewed, purposive sampling was used to recruit a diverse range of patients, in terms of age, sex, and smoking and employment statuses. The groups interviewed included 1) those who did not respond to the initial invitation to participate in the case-finding trial (nonresponders); 2) patients who returned a screening questionnaire that reported significant respiratory symptoms making them eligible for a spirometry appointment but DNA; 3) patients who attended spirometry and found not to have airflow obstruction or COPD (attendees without airflow obstruction [AO]); and 4) patients who attended spirometry and met the study criteria for a new diagnosis of COPD (COPD positive). Selected patients were sent further information about the interview and given the option of a face-to-face or telephone interview. Written informed consent was obtained from the patients attending the face-toface interviews. For the telephone interviews, patients were sent an informed consent form, which the interviewer read over the phone, covering each item and verifying that the patient was happy to go forward with the interview, documenting this on the researcher copy of the informed consent form, in accordance with normal practice. ${ }^{14}$

\section{Interviews}

Semistructured interview schedules used open-ended questions to explore patients' views on COPD case-finding in general and their perspectives on the individual processes involved, which were relevant to their experiences. All patients were asked about their recollections of the initial screening questionnaire. Those who were invited for a spirometry assessment were asked about their experience of receiving the invitation, their feelings toward the assessment, and their views on the process of receiving feedback 
of results. Finally, participants were asked to comment and reflect on any subsequent lifestyle behavior changes that they had made as a result of being invited for screening. Patients who were COPD positive had additional questions relating to the processes and experiences around diagnostic outcomes. Patient's perceptions on their health status and symptoms were explored, using open-ended questions. Interviews were carried out by one of the four trained research fellows. All interviews, except two which faced technical difficulties, were audio recorded and transcribed verbatim. Field notes alone were used for the remaining two patient interviews. Interviews were face-to-face, in the patient's home or by telephone, based upon patient's preference and lasted on average between 15 and 40 minutes, with a median of 35 minutes. Three postdoctoral researchers and one $\mathrm{PhD}$ student performed the interviews. The interviews were between one researcher and each participant; no additional people were present.

\section{Analysis}

Interviews were analyzed using the framework method. ${ }^{15}$ In accordance with this method, transcripts were read by KJ, SG, PA, RJ, and AE to identify codes and themes referring to specific steps in the screening process. A total of eight transcripts, two transcripts from each of the four main groups, were independently coded and compared by two investigators, to create the coding framework in Microsoft Excel. Coding and indexing of all subsequent transcripts were performed by AE. Emergent themes were presented and agreed by consensus. Codes and themes of the 43 interviews were systematically integrated into the framework. In the analysis phase, we compared and contrasted common themes across the entire sample and sought patterns and meanings. Data saturation across the sample was reached after 43 interviews were carried out, and no new themes arose from the data. ${ }^{16}$ A further 15 people who had agreed to potential interviews were then thanked and informed that there was no need for them to participate.

\section{Results}

\section{Participant recruitment}

Details of participant recruitment are summarized in Figure 1. A total of 751 patients were invited, 58 patients agreed, of whom 43 patients were interviewed. Patients from the four main categories reflecting key steps in the screening process were included.

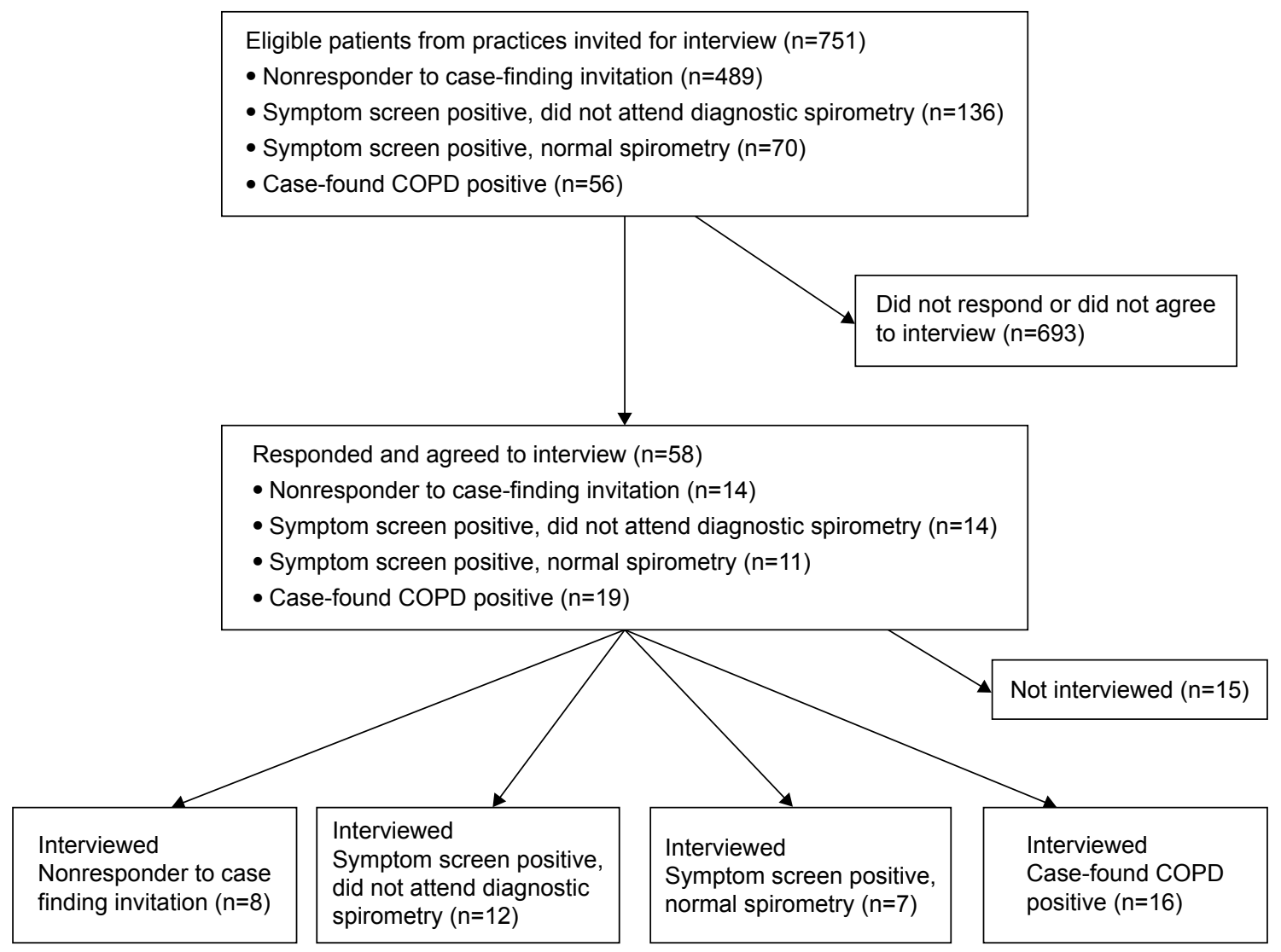

Figure I Recruitment flow diagram. 
Table I Characteristics of patients interviewed

\begin{tabular}{|c|c|c|c|c|}
\hline Characteristics & $\begin{array}{l}\text { Nonresponder } \\
(n=8)\end{array}$ & $\begin{array}{l}\text { Did not attend } \\
\text { spirometry } \\
\text { appointment }(n=\mid 2)\end{array}$ & $\begin{array}{l}\text { Symptomatic } \\
\text { without airflow } \\
\text { obstruction }(n=7)\end{array}$ & $\begin{array}{l}\text { COPD } \\
\text { positive } \\
(N=16)\end{array}$ \\
\hline Age (mean years) & 69 (6.8 SD) & $62(10.0 \mathrm{SD})$ & $66(1 \mathrm{I} .1 \mathrm{SD})$ & 66 (8.9 SD) \\
\hline Gender male & $6(75 \%)$ & $7(58 \%)$ & $4(57 \%)$ & $9(56 \%)$ \\
\hline Current smokers & 2 & 2 & 5 & 6 \\
\hline Unemployed or retired & 7 & 7 & 6 & 12 \\
\hline Early retirement (health reasons) & I & 3 & 2 & 1 \\
\hline In work & 0 & 5 & I & 4 \\
\hline
\end{tabular}

Fifteen patients had agreed to take part in the study but were not interviewed, as theme saturation had been reached. They had similar characteristics as the interviewees; however, there were $6 \%$ more males in this group.

\section{Patient characteristics}

Overall, the mean age of the participants was 66 years (SD 9.6 years); $60 \%$ were male, $37 \%$ were current smokers, and $23 \%$ were in work, at the time of the interview. The characteristics of the four individual screening groups are listed in Table 1.

There was one patient who reported never smoking, although their general practice records had suggested a positive smoking history, which had led to their inclusion in the trial.

\section{Main findings}

There were two main themes that evolved from the data: 1) patients' views on case-finding and related case-finding processes and 2) motivation to participate and barriers to successful case-finding (Figure 2). These themes are presented

Views on case-finding

\section{Processes}

How to case-find for COPD (who, time \& place)

- Acceptability of screening questionnaire

- Views on spirometry test

- Lack of feed back of spirometry results

- Perception of COPD+ diagnosis

- Nonresponders: why no response?

- Did not attend group: why not attend for spirometry?

\section{Motivation}

- Participation in research, to help others

- Patient symptomatic, wanted to know if anything was wrong sequentially below with illustrative quotes to summarize the range of views expressed. We have not used numerical values to describe the frequency with which themes were discussed.

\section{Patients' views on case-finding and its constituent processes}

Patients' perceptions on the specific steps in the screening process were identified across all groups in the analysis. Each major step of the process was explored: the acceptability of the screening questionnaire, the experience of spirometry, and any subsequent follow-up with the general practitioner. Overall, across the four groups of respondents, case-finding was deemed important and beneficial, as it was perceived to allow early identification and treatment of disease. Many used cancer screening as a comparator. Interestingly, one patient who had not returned the case-finding questionnaire stated:

I believe, as I said, case-finding has got to be good. I can't see any logical reason for not case-finding. [Nonresponder, 67-year-old male]

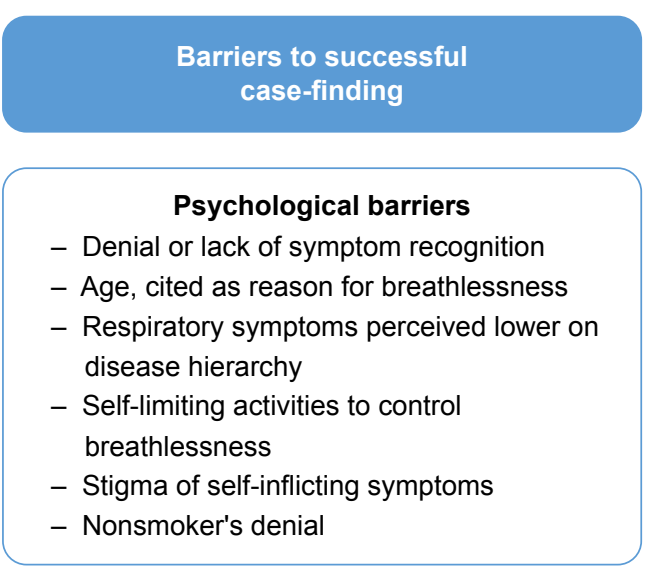

\section{Mechanical barriers}

- Too busy to participate (carer for other family member)

- Working, limited time

- Limited GP access, GP too busy

- Lack of diagnosis, feedback from screening

Figure 2 Patients' views on COPD case-finding, barriers to successful case-finding and related sub-themes. Abbreviation: GP, general practitioner. 
A patient subsequently diagnosed with COPD said:

I was very pleased to be honest, because I've been going to my doctors for eight years about this, and they don't do anything about it, and well, I thought "well, I'll go through this and then maybe they'll sort it out." [COPD-positive, 69-year-old female]

\section{The screening questionnaire}

There were no concerns about the screening questionnaire or the way it was administered, and patients generally commented on the ease of completing it. Among nonresponders, some explained that they completed the questionnaire, but despite the inclusion of a postage-paid envelope and two reminders, they did not send it back, citing forgetfulness or being too busy.

\section{Implementation of case finding}

Participants suggested that screening activities should take place in a convenient place, such as their general practice, as it is close to home and travel costs are minimal. In contrast, several patients felt that general practitioners had no time for screening, and difficulties accessing appointments in primary care is a barrier. Pharmacists were suggested as an alternative for implementing case-finding, as it was thought that they would have more time and were better at explaining things

... perhaps pharmacists might be a better option than doctors. If you ask for a specific type of drug or you've got a prescription for a specific type of drug, or you go in and say, "Ive got shortness of breath, what can you recommend?" Perhaps a pharmacist might be able to say, "Do you mind answering a few questions?" And you might get a better response there, because as I say doctors will say they haven't got the time, whereas pharmacists ... my pharmacist seems to be questioning about everything I go in there for. [Nonresponder, 62-year-old male]

Many participants commented on the importance of flexibility in the timing of case-finding assessments, as limiting this to 9 am to $5 \mathrm{pm}$ would not allow working people to participate. Misunderstanding of spirometry testing and fear of the procedure had prevented some participants from attending. This occurred despite a letter and a phone call explaining the procedures. Several patients complained that spirometry was exhausting and difficult to perform. Nevertheless, all these patients said that they would repeat it if needed. Overall, respondents felt that the effort of performing spirometry did not represent a substantial barrier.
The blowing test. It was alright. I don't think I did very well on the blowing test. I did find it hard work, yes., I did complete it. But I had to do it more than once, because the first time I couldn't do it. I think it was about the third time that we did it on. I would do it again, yes. [Symptomatic without airflow obstruction, 72-year-old male]

\section{Motivation to participate in case-finding}

Those who had taken up the invitation for case-finding primarily reported that they were motivated by altruistic reasons, to "do their bit for mankind", as this was a research study. However, some patients experiencing symptoms wanted to know if there was anything wrong. Others said that they had been smokers, so they thought that they had better have their lungs checked.

I really didn't have any breathing, lung problems, but I had this congestion which goes into the lungs and that, and that's what was worrying me, quite honestly, and that now the doctor treats me so differently, because I was (screened) ... I had got nowhere before [COPD-positive, 69-year-old female]

\section{Barriers to case-finding}

Although patients viewed case-finding as fundamentally desirable, there were numerous and diverse barriers that fell into the following two main categories: psychological and mechanical. Psychological barriers included denial of the diagnosis or failure to recognize symptoms, stigma of self-inflicted disease, fear of the "test", and lung symptoms being low on the hierarchy of patient health complaints. Mechanical barriers included providing care for another person (too busy), being unable to access general practice appointments, and lacking feedback of spirometry results or communication of the diagnosis.

\section{Psychological barriers}

Denial of the diagnosis or not recognizing symptoms was the most common theme that emerged throughout all four groups of patients, and there were several reasons offered to explain this.

Age: Many perceived that older age was the reason for their breathlessness.

I am more breathless than I used to be when I was younger. But it is hard to tell whether that's my age or whether it is any problem I have with the chest really, because I haven't been 71 before ... Whether it's age I am not sure [COPDpositive, 70-year-old male] 
I feel my knees affect my way of life more than my breathing, you see I did say ... somebody said "well, do you get out of breath?" and I said "Well, don't all people in their seventies?'.... So I've always took it as an aging process, you know you learn to walk slow ... [COPDpositive, 78-year-old female]

Self-limiting activities: The majority of patients denied problems with their lungs and, when probed further, reported self-limiting their activities to control their breathlessness.

If I start (walking) any incline or put any pressure on ... see what I tend to do is limit myself to within my capabilities, so if I feel myself getting short of breath I stop what I am doing because I don't want to get into that situation [Nonresponder, 62-year-old male]

\section{Hierarchy of comorbidities}

When asked about their general health status, patients often reported other significant comorbidities, which were of higher concern. All of the nonresponders reported moderateto-severe co-morbidities, including cancer, diabetes, angina, and other cardiovascular illnesses.

Patients reported adapting to dyspnea by self-limiting their activity. When asked if they had "chest or breathing problems", many patients denied experiencing any. However, when asked if they became breathless going up stairs, they reported activity avoidance, which seemed to reinforce their perception that they had no lung problems. As many patients self-limited their activity to control their disease symptoms, they did not perceive that the problems with their breathing were a major concern, compared to their other conditions.

I've had endocarditis, which I've had surgery last year, that's why I am in the state I' $m$ in now with vertigo ... I've got MS ... I suffer from tinnitus and have done so for years, and I get the usual aches and pains.... But other than that my general health is quite good [Nonresponder, 67-yearold male]

\section{Lack of confidence in health care services or previous} negative health care experience

Several patients reported having negative experiences with health services. One common complaint was the perceived lack of general practitioner initiative for managing their long-term symptoms, despite multiple visits. Some patients felt that general practitioners were apathetic or unaware of what was ailing them. One patient, subsequently diagnosed with COPD, reported that she had been attending her general practice for 8 years with the same complaint and felt that her complaints had been untreated. Another patient who had also reported long-term respiratory problems said:

to be honest with you, I think they had given up on me [Spirometry appointment nonattender, 59-year-old male]

I can't turn around and say "well I think I've got this ...", and he could turn around and say "well hang on, I'm the doctor, I'll tell you what's wrong with you" which they do. But all it is, you go in and all they do is examine you, you know, "here's your prescription and ta-rar, see you again" ... They want to get you in as soon as they can and get you out straight away. [Nonresponder, 67-year-old male]

A small proportion of patients felt that there was stigma and blame attached to smoking and that the health service discriminated against smokers.

I know that the end of it, it's my own fault ... if I've smoked and I get something from it, I know its my own fault and obviously the national health is there to help everybody but I understand if you've done something that you shouldn't be really doing then they're not going ... well, how can I put it, they're not going to treat you, I don't think, the same as they would somebody who hadn't ever done something they shouldn't have done in life. [COPD-positive, 71-yearold female]

\section{Fear of testing/results and fear of diagnosis}

A small number of patients expressed fear of the case-finding process or of receiving a diagnosis of COPD, but the majority felt that it was best to find out if they had a problem.

Why not go for the test or the case-finding and see what happens, and hopefully it will come back that I'm fine... . I wanted to do it, more for peace of mind. [Spirometry appointment nonattender, 57-year-old male]

Conversely, one patient who had recently been diagnosed with COPD through case-finding expressed some regret in having participated.

I would never have sort of diagnosed myself as having that kind of problem so ... I think that's just the downfall that I, I agreed to take part. [COPD-positive, 67-year-old male]

\section{Mechanical barriers}

\section{Too busy and other priorities}

There were many patients who were care-givers for sick spouses or chronically ill family members. They felt that they did not have the time to pay attention to their own 
health issues, despite the offer of case-finding provided in their local general practice. When one nonresponder was asked if they would have come to their general practice for spirometry, he replied:

No, not really, it would depend on how long the test would take... . Because I would have to come and see you people, it's leaving the wife because it's a long time because she's disabled you see.... Well yes, yes, my health is one ... but the way the wife is, she comes first. She's top priority. [Nonresponder, 67-year-old male]

\section{General practitioner too busy and limited access to appointments}

Patients felt that a substantial barrier to successful casefinding was limited access to general practice appointments. They expressed doubt that general practitioners would have adequate time to perform case-finding.

... We've got a doctor down the surgery ... and you can try phoning for 3 months to try and get an appointment with him

[Spirometry appointment nonattender, 65-year-old male]

\section{Lack of diagnosis and lack of feedback of spirometry test results}

Of the 23 participants who had attended for spirometry, 17 participants were COPD positive, but only four participants had received a diagnosis by their general practitioner, 6-12 months after the results had been sent to their doctor. Among these four participants, two participants denied that their general practitioner had told them they had COPD, the third patient believed that they had been told they had "borderline" COPD, and the fourth patient had been sent for further investigations and offered smoking cessation but was not specifically told they had COPD.

She (the General Practitioner) said that they're requiring chest $\mathrm{x}$-rays so wanted to keep me in, have a chest $\mathrm{x}$-ray and I had the results in less than a week and they ... it was clear, they did all offer, to help to go to smoking cessation and I said "I'll think about it" because they always ask you that anyway, so I thought about it and I haven't done anything, but who knows, I mean, going through all of this, maybe I might take that up at some point. [COPD-positive, 65-year-old female]

A total of 13 of the 17 COPD-positive patients were not informed of their diagnosis. They reported that they assumed that there was nothing to worry about and they would have heard from their General Practitioner if they had COPD.
I was told no results at all. I have assumed because I have heard nothing everything is okay, but I have never had any results back via the doctor to say we've done these tests and you really need so and so, okay for your age, or whatever. So I have no results back in that sense from my doctor at all, no... No, nothing at all no, so I've just assumed everything is fine. I must admit I would have preferred the doctor to have had me in and said, "You had your breathing tests and they're fine." But he didn't, I have just assumed because he hasn't come back to me everything is okay. [COPDpositive, 70-year-old male]

\section{Discussion}

To our knowledge, this is the first study eliciting primary care patients' views and experiences on participation in case-finding for COPD and specific components of the process (invitation, the screening process, and diagnostic outcomes).

\section{Main findings}

While the majority of those interviewed found the concept of COPD case-finding acceptable, more than half $(n=20)$ had either not responded to the screening questionnaire or not attended spirometry when it was offered to them. A number of explanatory psychological and mechanical barriers were uncovered. Lack of knowledge about the condition, denial or failure to recognize indicative symptoms (through adaptive self-limiting of behavior or fear and stigma attached to the condition), and lower attention to COPD symptoms compared with those from other existing comorbidities contributed to this. These barriers were compounded by other obstacles such as difficulty accessing medical appointments and frustration with health services for not managing their ongoing symptoms.

With respect to the implementation of case-finding, participants who had undergone screening had found the process acceptable. However, many patients felt that general practitioners were too busy and lacked capacity to undertake this, either based on their own previous negative experience or due to feared stigma attached to smoking. These perceptions were borne out among COPD-positive patients, most of whom had not had their diagnosis confirmed by their doctors.

\section{Interpretation of findings in relation to previously published work}

Our finding that patients deny symptoms or do not recognize disease onset concurs with those from other studies, which focused on the diagnosis and management of COPD. These studies, including some from other countries and different 
health care systems, reported the attribution of symptoms to aging or smoking. ${ }^{4,6}$ Many patients do not perceive their respiratory symptoms to be related to a serious condition. Arne et $\mathrm{al}^{5}$ interviewed COPD patients at the time of diagnosis, which was not related to a case-finding program. They reported that the lack of knowledge of the implications of symptoms, feelings of shame, and lack of a clear diagnosis were barriers to managing COPD. In a qualitative study exploring reasons for COPD patients' low uptake of services, Gysels and Higginson ${ }^{12}$ found that, as in our study, symptoms such as breathlessness were not perceived to be serious and the adaptability of patients to manage their symptoms further masked this. Similar to our findings, Leidy et $\mathrm{al}^{6}$ described the denial or lack of attribution of symptoms, which was assumed to be caused by aging, smoking, weight, or other comorbidities.

We found as COPD progresses, patients become accustomed to self-limiting their activity, which disguises the gradual progression of disease, and patients perceive other health problems, such as cancer and diabetes, to be more of a day-to-day burden. In another study, Jones et a ${ }^{17}$ identified many missed opportunities to diagnose COPD in primary care due to a number of factors, including under-recognition of symptoms. This represents a significant barrier to the success of case-finding initiatives: patients may not engage with an invitation to participate until they have developed significant symptoms and they can no longer adapt activity levels.

Another barrier to the successful implementation of COPD case-finding we found was a perceived lack of clinical engagement in case-finding and giving patients a clear diagnosis of COPD. When general practitioners and health care workers were interviewed in a related study, primary care staff felt that patients often under-estimated the significance of their COPD symptoms or were not always forthcoming about them or about their smoking habits. ${ }^{18}$ In the current study, many of our participants perceived that general practitioners were inadequately responding to their symptoms that they found frustrating. Our study also found that 6-12 months after test results were sent to their doctors, the majority of patients interviewed had not received a COPD diagnosis. This concurs with findings from a qualitative study by Walters et al, ${ }^{4}$ which found that general practitioners were often reluctant to label the disease and, on average, delayed the diagnosis of COPD by $>5$ years. They reported that patients often received their COPD diagnosis as a result of a hospital visit, rather than in primary care. Exploration of the views of health care professionals involved in the case-finding trial highlighted limited capacity, resources, and expertise among primary care practitioners as potential explanations for this delay. ${ }^{18}$ A more recent UK study by Summers et al ${ }^{19}$ found that although health care professionals implementing targeted case-finding in primary care agreed that diagnosing COPD earlier had benefits, patients' increased stress and negative responses added more strain on limited resources.

Our study also identified that stigma, associated with being a smoker, could be a barrier to taking up a diagnostic assessment for COPD. The studies by Arne et $\mathrm{al}^{5}$ and Gysels and Higginson, ${ }^{12}$ cited previously, reported similar findings with some patients perceiving that health professionals might see their condition as self-inflicted and that this would bias the health care they would receive. Chapple et $\mathrm{al}^{20}$ reported that patients with a similar smoking-related disease, such as lung cancer, experienced stigma, shame, and blame for their disease. This stigma compelled patients to conceal their condition, which prevented them from receiving support, and could undermine case-finding efforts unless addressed.

\section{Strengths and limitations of this study}

This is the first study exploring patients' perceptions at each stage of the case-finding process in primary care. The study was designed to capture views of four different patient groups from those invited to participate. This allowed exploration of different barriers and enablers of the process and incorporation of the views of a wide range of patients who might be at risk of early-stage COPD. We did not invite people who responded that they had no chronic respiratory symptoms in the initial screening questionnaire for interview. Thus, the views of this group in relation to case-finding are not known and should be explored in future research. The timing of the qualitative study, 6-12 months after the spirometry results were sent to the general practice, should have allowed sufficient time for general practitioners to contact the COPDpositive patients. The sample size was sufficient to allow data saturation of themes.

Limitations of our study include the fact that patients interviewed had been invited in the context of a research study; so the views expressed may not necessarily reflect those of patients invited to participate in case-finding as part of a routine health service. The research was performed in a UK setting, where access to general practice appointments and quality spirometry may represent barriers; this may not be true in other primary health care systems. We did not repeat interviews or return the transcripts to the participants for their comments; this may have produced slightly different results. Recruitment of the general practices was limited to 
those who had sufficient time to provide patients with their screening results and may not be representative of the wider range of patients. Interviews were undertaken either faceto-face or by telephone, depending on patient preference. This may have elicited different responses from patients. However, another study that compared findings from interviews conducted using each of these methods found that the emerging themes did not differ between methods. ${ }^{21}$ Finally, the analysis and interpretation of the qualitative data may have been influenced by the prior beliefs of the investigators who are involved in a large program of research on the early detection of COPD. However, the initial themes for this qualitative study were developed by a multidisciplinary group and by people who had different levels of involvement in the main trial.

\section{Implications for future research, policy, and practice}

COPD will become a greater burden on the National Health Service (NHS) as the population ages. Improving care and quality of life for patients with COPD may be facilitated by earlier diagnosis, yet a number of patient and health servicerelated factors may prevent this. The public and health care professionals should be trained to recognize the early symptoms of COPD, particularly breathlessness. Case-finding services should ideally be available in the community, including general practices and community pharmacies at times to enable those in work to attend, and possibly incorporated into the management of other chronic diseases. An efficient process to ensure whether patients receive their results in a timely manner needs to be in place; this has been reported in other settings where case-finding has occurred. Based on our findings, timely feedback and intervention are important. General practitioners need to counsel patients openly about their diagnosis and lifestyle behaviors and offer reassurance that patients are not being judged or discriminated against with respect to their smoking habits; this perception of admonishment has been found in other health care system settings, as mentioned earlier. Primary care must also have increased capacity to properly manage the growing number of patients with COPD. Finally, additional work should be done to examine the long-term benefits of diagnosing COPD on patients' subsequent health behaviors (eg, smoking cessation), COPD management, and health outcomes.

\section{Conclusion}

The patients interviewed believed that case-finding for COPD is important, although there are a number of barriers to engaging with this process. Patient engagement with case-finding may be limited by denial or lack of recognition of symptoms, and the decision to take up screening may be aided by increasing public awareness of COPD risk factors and early symptoms. Health care professionals may benefit from training to clearly communicate the diagnosis of COPD to patients in a timely manner. Smokers should be reassured that they will receive nonjudgmental care. If case-finding is to be successfully implemented, logistical barriers to participation must be addressed by providing flexible appointment times at convenient locations.

\section{Acknowledgments}

Many thanks, to Dr Joanne O’ Beirne-Elliman, for coordinating this study. We thank all the participants interviewed. We thank the General Practices and our patient advisory group for their useful comments. We thank the Clinical Research Network for recruitment, and finally, we thank the BLISS research team for their hard work in making this trial possible. SG and KJ are partly funded by the National Institute for Health Research (NIHR) Collaboration for Leadership in Applied Health Research and Care West Midlands (CLAHRC WM). The views expressed are those of the authors and not necessarily those of the NIHR, the NHS, or the Department of Health. This article summarizes independent research funded by the NIHR under its Programme Grants for Applied Research Programme (Grant Reference Number RP-PG-0109-10061). KJ is partly funded by the NIHR CLAHRC West Midlands. The views expressed are those of the authors and not necessarily those of the NHS, the NIHR, or the Department of Health. TargetCOPD is a part of The Birmingham Lung Improvement StudieS - BLISS. The members of the BLISS research team were as follows: P Adab, KK Cheng, BG Cooper, A Daley, AP Dickens, J O’Beirne-Elliman, A Enocson, D Fitzmaurice, S Greenfield, S Haroon, K Jolly, R Jordan, S Jowett, K Kalirai, J Marsh, MR Miller, R Riley, R Stockley, and AM Turner.

\section{Author contributions}

The concept of a need for the trial was identified by REJ/PA, and the design was refined in discussion with DF. PA and REJ, with support from SG and KJ, designed the qualitative element. SG advised on analysis. AE undertook the qualitative analyses with guidance from $\mathrm{KJ}$ and $\mathrm{SG}$. $\mathrm{AE}$ wrote the first draft of this article with substantial contributions from SG, KJ, PA, and REJ. All authors had input to the design and contributed and approved the final article. All authors contributed toward data analysis, drafting and 
revising the paper and agree to be accountable for all aspects of the work.

\section{Disclosure}

PA, REJ, and KJ report grants from NIHR. The other authors report no conflicts of interest in this work.

\section{References}

1. Lozano R, Naghavi M, Foreman K, et al. Global and regional mortality from 235 causes of death for 20 age groups in 1990 and 2010: a systematic analysis for the Global Burden of Disease Study 2010. Lancet. 2012;380(9859):2095-2128.

2. Wedzicha JA, Wilkinson T. Impact of chronic obstructive pulmonary disease exacerbations on patients and payers. Proc Am Thorac Soc. 2006;3(3):218-221.

3. Ford ES, Mannino DM, Wheaton AG, Giles WH, Presley-Cantrell L, Croft JB. Trends in the prevalence of obstructive and restrictive lung function among adults in the United States: findings from the National Health and Nutrition Examination surveys from 1988-1994 to 2007-2010. Chest. 2013;143(5):1395-1406.

4. Walters JA, Hansen EC, Walters EH, Wood-Baker R. Under-diagnosis of chronic obstructive pulmonary disease: a qualitative study in primary care. Respir Med. 2008;102(5):738-743.

5. Arne M, Emtner M, Janson S, Wilde-Larsson B. COPD patients perspectives at the time of diagnosis: a qualitative study. Prim Care Respir J. 2007;16(4):215-221.

6. Leidy NK, Kim K, Bacci ED, et al. Identifying cases of undiagnosed, clinically significant COPD in primary care: qualitative insight from patients in the target population. NPJ Prim Care Respir Med. 2015;25:15024.

7. Roberts J. COPD Screening and Case Finding. Primary Care Respiratory Society UK; 2010:1-2. Opinion No. 38. Solihull, West Midlands.

8. Au DH. Screening for chronic obstructive pulmonary disease: $\mathrm{D}$ is the new F. JAMA Intern Med. 2016;176(5):601-602.

9. Chronic obstructive pulmonary disease in adults. Quality Standard. Published by the National Institute for Health and Care Excellence, London; 2011:50.
10. Siu AL, Bibbins-Domingo K, Grossman DC, et al. Screening for chronic obstructive pulmonary disease: US Preventive Services Task Force recommendation statement. JAMA. 2016;315(13):1372-1377.

11. Team KHDoHMDR. An Outcomes Strategy for Chronic Obstructive Pulmonary Disease (COPD) and Asthma. England: Department of Health; 2011.

12. Gysels M, Higginson IJ. Access to services for patients with chronic obstructive pulmonary disease: the invisibility of breathlessness. J Pain Symptom Manage. 2008;36(5):451-460.

13. Jordan RE, Adab P, Jowett S, et al. TargetCOPD: a pragmatic randomised controlled trial of targeted case finding for COPD versus routine practice in primary care: protocol. BMC Pulm Med. 2014;14:157.

14. Irvine ANESRC. Research Methods: Using Telephone Interviews. Research Methods: Toolkit 14. University of York: National Economic \& Social Research Council, Realities, Morgan Centre University of Manchester; 2010:7.

15. Gale N, Heath G, Cameron E, Rashid S, Redwood S. Using the framework method for the analysis of qualitative data in multi-disciplinary health research. BMC Med Res Methodol. 2013;13:117.

16. Guest G, Bunce A, Johnson L. How many interviews are enough? An experiment with data saturation and variability. Field methods. 2006; 18(1):59-82.

17. Jones RC, Price D, Ryan D, et al. Opportunities to diagnose chronic obstructive pulmonary disease in routine care in the UK: a retrospective study of a clinical cohort. Lancet Respir Med. 2014;2(4): 267-276.

18. Haroon S, Jordan RE, Fitzmaurice DA, Adab P. Case finding for COPD in primary care: a qualitative study of the views of health professionals. Int J Chron Obstruct Pulmon Dis. 2015;10:1711-1718.

19. Summers RH, Sharmeen T, Lippiett K, et al. A qualitative study of GP, nurse and practice manager views on using targeted case-finding to identify patients with COPD in primary care. NPJ Primary Care Respir Med. 2017;27(1):49.

20. Chapple A, Ziebland S, McPherson A. Stigma, shame, and blame experienced by patients with lung cancer: qualitative study. BMJ. 2004; 328(7454): 1470.

21. Sturges J, Hanrahan KJ. Comparing telephone and face-to-face qualitative interviewing: a research note. Qual Res. 2004;4(1):107-118.
International Journal of COPD

\section{Publish your work in this journal}

The International Journal of COPD is an international, peer-reviewed journal of therapeutics and pharmacology focusing on concise rapid reporting of clinical studies and reviews in COPD. Special focus is given to the pathophysiological processes underlying the disease, intervention programs, patient focused education, and self management protocols.

\section{Dovepress}

This journal is indexed on PubMed Central, MedLine and CAS. The manuscript management system is completely online and includes a very quick and fair peer-review system, which is all easy to use. Visit $\mathrm{http} / / / \mathrm{www}$.dovepress.com/testimonials.php to read real quotes from published authors. 